\title{
Emergency Room
}

National Cancer Institute

\section{Source}

National Cancer Institute. Emergency Room. NCI Thesaurus. Code C53513.

The section of a hospital or clinic staffed and equipped to provide immediate medical treatment. 